\title{
Network-Centric Control Methods for a Group of Cyber-Physical Objects
}

\author{
Vladimir Muliukha ${ }^{1} \quad$ Alexey Lukashin $^{2} \quad$ Alexander Ilyashenko $^{2} \quad$ Vladimir Zaborovsky $^{2}$ \\ ${ }^{1}$ Almazov National Medical Research Centre, St.Petersburg, Russia, mulyukha_va@almazovcentre.ru \\ ${ }^{2}$ Peter the Great St. Petersburg Polytechnic University, St.Petersburg, Russia, lukash@ @eva.ru, \\ ilyashenko.alex@gmail.com,vlad@neva.ru
}

\begin{abstract}
In the paper we propose to use network-centric approach for a control task. Robots are described as cyber-physical objects that consist of two parts: mechatronic and informational. All cyber-physical objects are connected with each other using special multiprotocol nodes - devices that can route data between different types of computer networks (Ethernet, WiFi, 3G, LTE). Such network is described by hypergraph model, where central node is a hybrid cloud computer. While all robots are connected together, logical and computational tasks for cyberphysical objects are processed by this high performance node like in the central control system. Without a connection with central node robot switches into a multiagent mode.
\end{abstract}

Keywords: cyber-physical object, network-centric control, cloud robotics

\section{Introduction}

Modern trends in industrial technology include theoretical and applied research aimed at finding effective methods to control distributed dynamic systems. This research has particular importance for scientific foundations of field robotics that were greatly improved in recent years by the cyber-physical ideas. In this paper we provide constructive analysis focused on possibilities of application of cyber-physical approach to robots' motion planning and coordination to achieve control objectives in spatially and temporally undefined conditions. Proposed approach is based on multi-invariant actor-information representation and cooperative interaction of all components that describes a robotics system both on the physical (local or actor-based) and informational (knowledge or ontological-based) levels which are implemented using distributed resources of private cloud computing environment as IaaS and Hadoop. Actor-based representation (model) of each physical objects or artificial machine (robot) has specific attributes including name, data stack and parameters. The model describes environmental characteristics of mechanical, sensor, navigation and computercommunication components, the local interaction of which takes place onboard the robot and is needed to achieve declared control objectives. An informational model is proposed to represent common system characteristics, objective features, and robot as mobile carrier of specific operations. It is shown that system control requirements may be reduced to a "constraint satisfaction" problem. The decision of such problem is expressed by two sets of entities: a set of operations performed by robots of the group, and a set of messages that are generated by the informational model and delivered to each robots of the group using network infrastructure.

\section{Cyber-Physics Approach}

Complex engineering tasks concerning control for groups of mobile robots are not yet sufficiently developed. In our work, we use cyber-physical approach, which extends the range of engineering and physical methods for a design of complex technical objects by researching the informational aspects of communication and interaction between objects and with an external environment.

It is appropriate to consider control processes with cyber-physical perspective because of the necessity for spatio-temporal adaptation to changing goals and characteristics of the operational environment. Thus the priority task is to organize the reliable and highperformance system of information exchange between all entities involved in the realization of all requirements. Hereinafter, by cyber-physical object we mean an open system for the information exchange processes. Data in such system is transmitted through the computer networks, and its content characterizes the target requirements achieved through execution of physical and mechanical operations, energy being supplied by the internal resources of the object (Figure 1).

An example of a cyber-physical object is a mobile robot that does complex spatial movement, controlled by the content of the received information messages that have been generated by a human-operator or other robots that form a multi-purpose operation network. An ontological model of informational open cyberphysical object may be represented by different formalisms, such as a set of epistemic logic model 
operations parameterized by data of local via computer connection. measurements or messages received from other robots

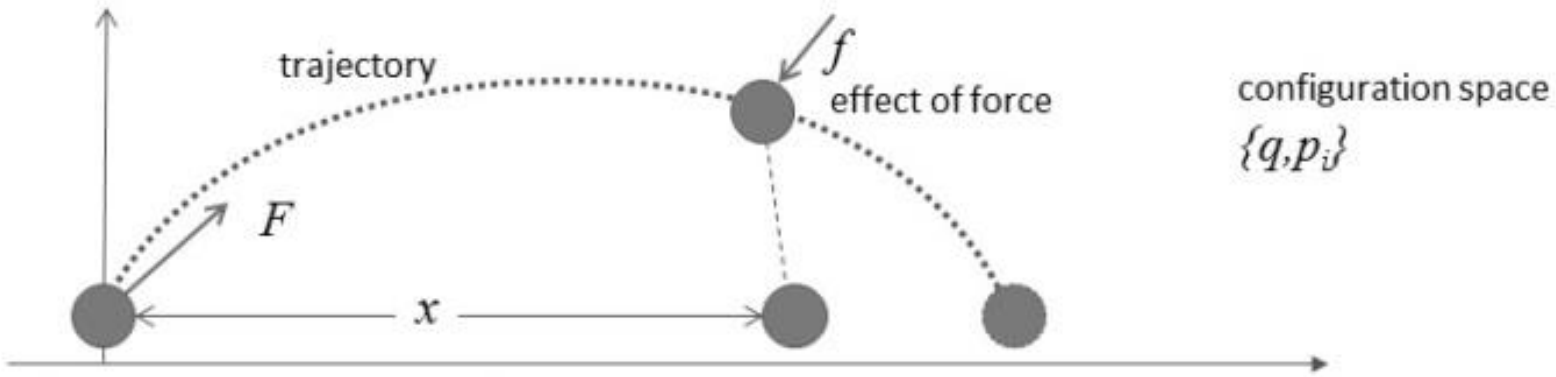

Physics as a science of causality:

movement trajectory of the object is changed by external «force»

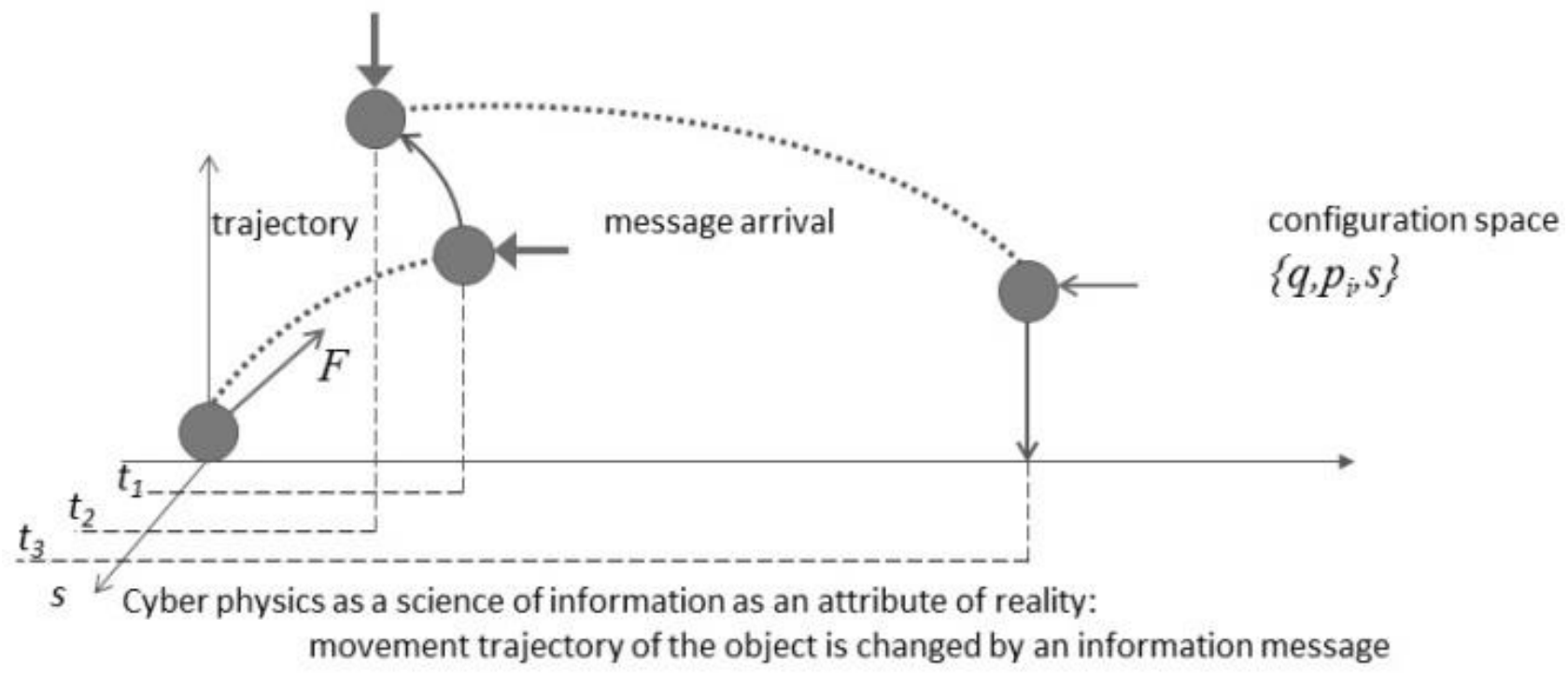

Figure 1. Physical and cyber physical motion

According to Figure 2, cyber-physical model of control system can be represented as a set of components, including following units:

- information about the characteristics of the environment (Observation),

- analysis of the parameters of the current state for the controlled object (Orientation),

- decision-making according to the formal purpose of functioning (Decision),

implementation of the actions that are required to achieve the goal (Action).

The interaction of these blocks using information exchange channels allows us to consider this network structure as a universal platform, which allows us to use various approaches, including the use of algorithms and feedback mechanisms or reconfiguration of the object's structure for the goal's restrictions entropy reduction or the reduction of the internal processes' dissipation.

Centralized solutions allow using universal means for the organization of information exchange to integrate different technologies for both observed and observable components of the controlled system. The parameters and the structure of such control system can quickly be adjusted according to the current information about the internal state of the object and the characteristics of the environment, which are in a form of digital data. These features open up the new prospects for the development of intelligent cyber physical systems that will become in the near future an integral part of the human environment in the information space of so-called "Internet of Things". According to the estimates (Zaborovsky et al., 2014), network-centric cyber-objects in the global information space of the Internet will fundamentally change the social and productive components of people's lives. 


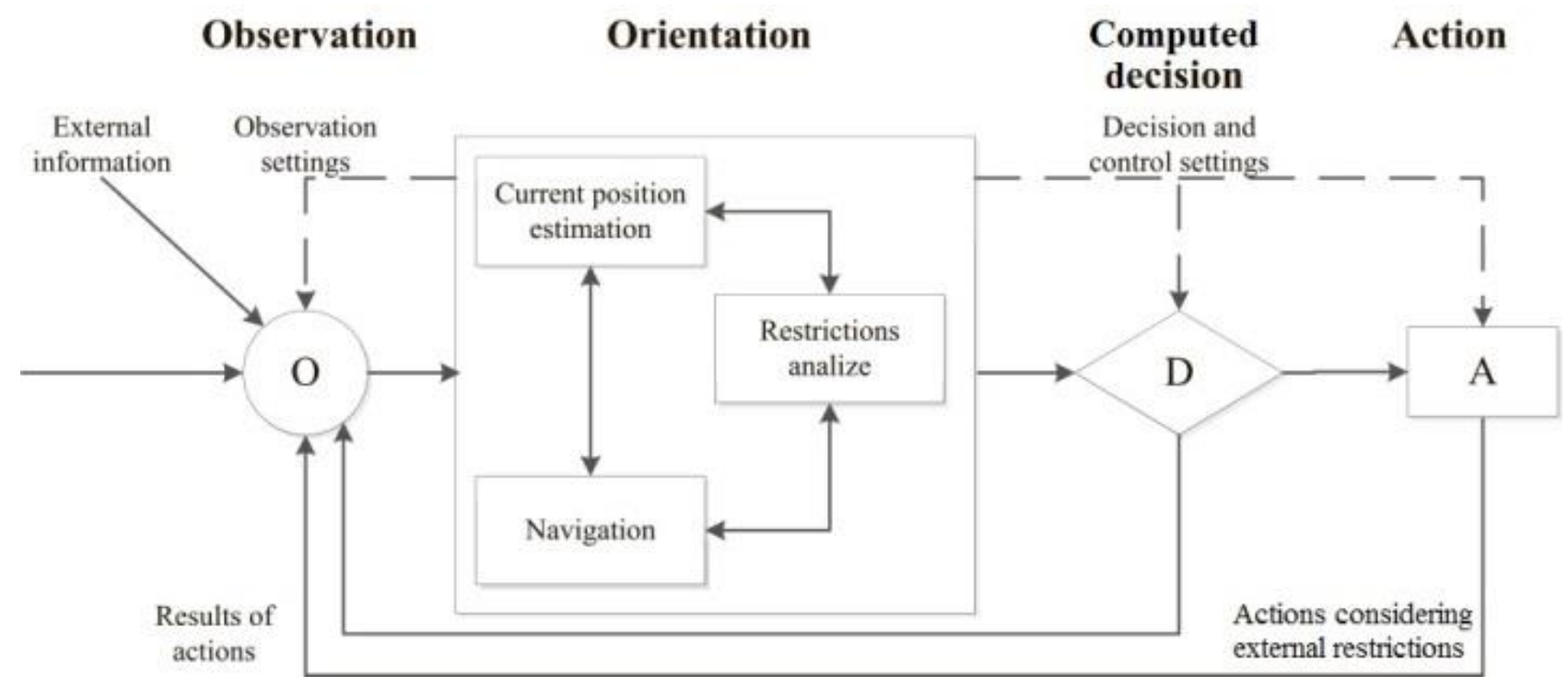

Figure 2. Cyber-physical interpretation of the John Boyd's OODA loop

The selection of cyber physical systems as a special class of designed objects is due to the necessity of integrating various components responsible for computing, communications and control processes («3C» - computation, communication, control). Therefore, the description of the processes in such systems is local and the change of its state can be described by the laws of physics, which are, in its most general form, a deterministic form of the laws of conservation of, for example, energy, mass, momentum, etc. The mathematical formalization of these laws allows us to determine computationally the motion parameters of the physical systems, using position data on the initial condition, the forces in the system and the properties of the external environment. Although the classical methodology of modern physics, based on abstraction of "closed system" is significantly modified by studying the mechanisms of dissipation in the so-called "open systems", such aspect of reality as the information is still not used to build the control models and to describe the properties of complex physical objects. In the modern world, where the influence of the Internet, supercomputers and global information systems on all aspects of the human activity becomes dominant, accounting an impact of information on physical objects cannot be ignored, for example, while realizing sustainability due to the information exchange processes. The use of cyber physical methods becomes especially important while studying the properties of systems, known as the "Internet of Things", in which robots, network cyberobjects and people interact with each other by sharing data in the single information space for the characterization of which are used such concepts as "integrity", "structure", "purposeful behavior", "feedback", "balance", "adaptability", etc.

The scientific bases for the control of such systems have become called Data Science. The term "Big Data" describes the process of integration technologies for digital data processing from the external physical or virtual environment, which are used to extract useful information for control purposes. However, the realization of the Data Science potential in robotics requires the creation of new methods for use of the information in control processes based on sending data in real time at the localization points of moving objects (the concept of "Data in motion").

\section{Heterogeneous Platform for Cloud Robotics}

The priority field of the development of science and technology are information and communications technologies and telematics services that are the foundation of modern infrastructure of the supercomputing engineering platform (Zaborovsky et al., 2014). The effectiveness of such a platform is ensured by cloud computing services that are implemented on the basis of hybrid supercomputing systems to effectively solve actual scientific and technical problems, including the following tasks: control of cyber-physical objects, predictive modeling and information security. The purpose of this paper is to solve the problem of the integration hybrid supercomputing resources in the control loop for a group of mobile robots, so called cyber-physical objects.

The base idea for the implementation of cloud technologies in the robotics is based on the paradigm of digital physics ("it from bit doctrine") (Wheeler, 1989), which implies that for robots the whole universe is measurable and computable. It means that if an object of the real "physical" world cannot be converted to the information from a sensor, for the robot and its control system such an object does not exist. So the key function of the robot's control system is to process 
data received from sensors into the commands for actuators of the robot. For a number of situations, when such processing should not be performed in real time, it can be performed remotely in the cloud. The application of cloud for the solution for modern robotics tasks is called "cloud robotics" (Kehoe et al., 2015). The use of this approach reduces the processing load on each robot in the group and increases the efficiency of all cyber-physical objects by increasing the duration of battery life and reducing the redundancy of robots' characteristics for its tasks.

Researches in the field of cloud technologies in robotics are relevant and have been particularly active in recent years. For example, Rapyuta platform should be noted (the RoboEarth Cloud Engine). It allows robots to share knowledge, through a centralized knowledge base, avoiding duplication of information. Rapyuta is a cloud platform, in which the robots can create their own computing environments to perform intensive data processing. These created environments may be used with one or more robots simultaneously. This work is realized using the cloud based on modified OpenStack platform that is installed on heterogeneous high-performance computers containing SIMD and MIMD processors.

In this work, we offer the following solutions: a central server located on a secure heterogeneous cloud receives data from sensors of all available cyberphysical objects (mobile robots), processes the data and commands from the operator, and sends control commands to cyber-physical objects. The developed cloud software performs a decomposition of the complex target, which is set by operator, into the simple operation, transmits them to mobile robots, and checks their implementation, as well as provides synchronization of commands execution when it is needed. The use of the heterogeneous computing environment allows effectively realize a wide range of tasks, including processing of streaming data from cyber-physical objects (e.g., video or lidar data). An actor approach resolves the problem of dividing a whole system into several parallel streams allowing the horizontal and vertical scaling of the developed system. A functional hierarchical scheme of cloud computing environment is shown in Figure 3.

As part of this work a following concept was developed: each robot or cyber-physical object consists of mobile hardware and software part, so called "agent" and virtual "avatar" represented by a set of computational processes that are implemented in heterogeneous cloud computing environment, and are interacting with the agent using wireless high-speed networks. Such operating model of cyber-physical objects, in which onboard computing resources are supplemented by cloud computing environment's services, can solve a variety of control tasks, and provide a number of important benefits, including:
- advanced class of algorithms for control and operations planning because of integrating all available information resources from cyberphysical objects,

- ability to store and to structure large volume of sensory data,

- common data space and situational awareness of all agents in the system,

- possibility of rapid change in avatar algorithms (reboot avatar of cyber-physical object in real time), interaction between avatars through the high-speed data network in the cloud environment.

\section{Practical Task: Constructing a Joint Map Task}

We've considered one of resource-intensive tasks for the robotic group control. This task is to build and maintain the relevance of the joint map of the surrounding environment. The appearance of a controlled object is shown in Figure 4. An example of the proposed solution is shown in Figure 5.

Data from the lidar comes into onboard computer, which attaches to it a timestamp and the current position obtained from the navigation system. The resulting data are pre-filtered and sent to a highperformance cloud environment for further processing, synthesis and storage. Joint point cloud is formed in heterogeneous high-performance cloud environment using received lidar data with a timestamp and coordinates:

- an area of the existing joint point cloud is selected using coordinate information;

- common fragments in new and existing data are allocated;

- calculated translation and rotation matrix for the new point cloud;

- new point cloud is merged with the existing one and the resulting area of the joint point cloud is filtered.

Further, a global joint point cloud received from all mobile cyber-physical objects is vectorized. A map marked-up for passability is formed for each of robots in terms of its characteristics. It is possible to consider not only geographically but also telematic terrain characteristics, for example, a connection availability in a certain area.

All action of mobile cyber-physical objects are planned in high-performance cloud environment using marked-up maps. For example, when the operator sets the end point for the mobile robot, the route is laid within the permissible area defined by the marked-up map. During the movement of the robot the global joint point cloud and the map based on it are constantly updated. In this case, if the route goes beyond the 
known area it will be corrected while new point cloud data will appear.

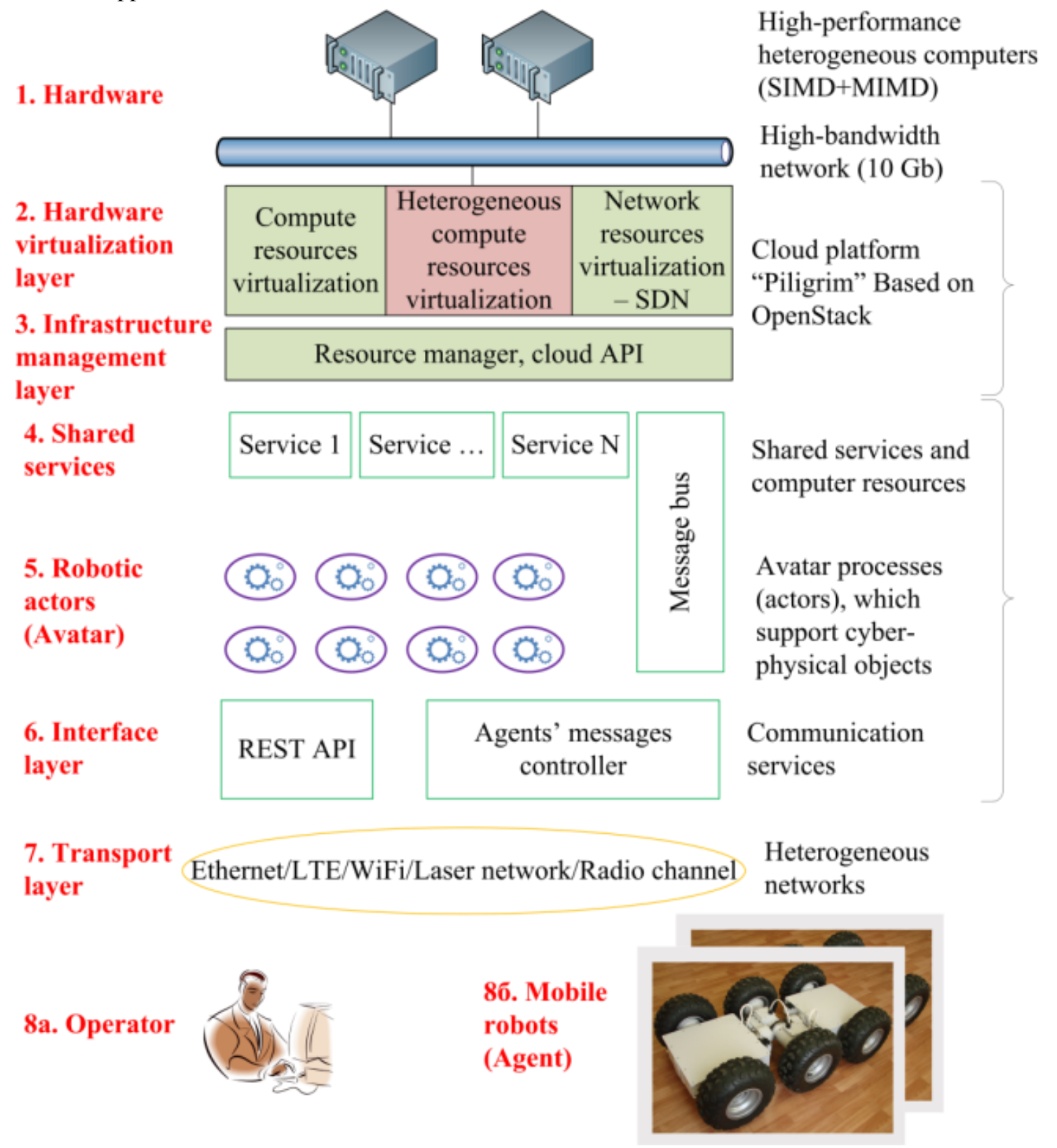

IaaS

PaaS

Figure 3. A functional hierarchical scheme of cloud computing environment

A fragment of generated route is transmitted to the onboard computer, which carries out the local control of the deviation between the current position and the desired trajectory. When the desired trajectory cannot be realized, the onboard computer requests new data from the high-performance computing environment.

In this work we use a point cloud library (PCL) and apply its existing methods. So one of the possible methods for point cloud vectorization is a mesh triangulation.

On the first step the point cloud is filtered to reduce the load on the compute nodes. Next, the triangulation is performed on the basis of data obtained. Depending on the degree of filtration it is possible to reduce the amount of data in more than ten times, while retaining sufficient accuracy of the model. An example of the proposed vectorization is shown in Figure 6.

\section{Information Exchange in Network- Centric Control System}

Main purpose of computer networks in network-centric control system is to transfer data between mobile robots (agents) and their virtual avatars. For working out effective transfer methods it is necessary to represent, what is the network, what processes proceed 
in it and what influences its performance. To answer these questions it is necessary to develop a network model.

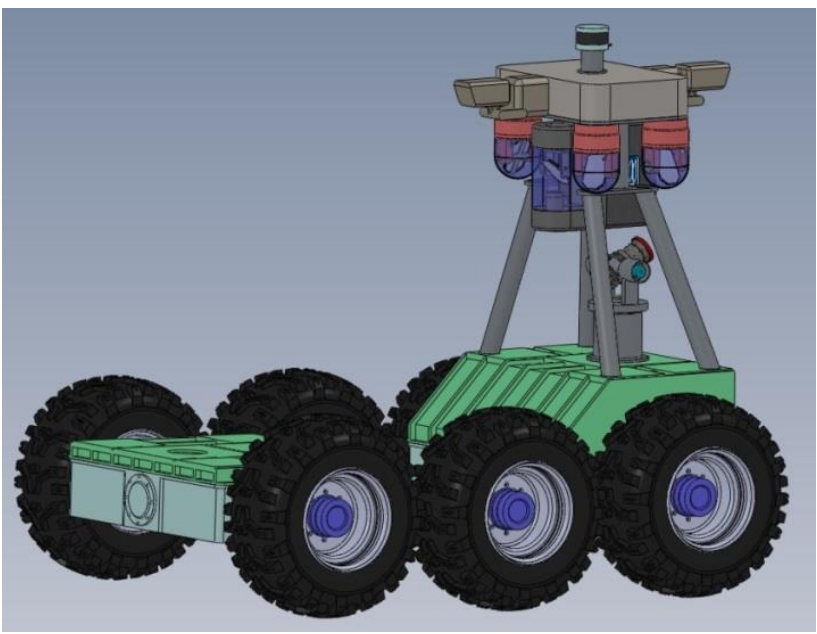

Figure 4. Appearance of controlled mobile robot

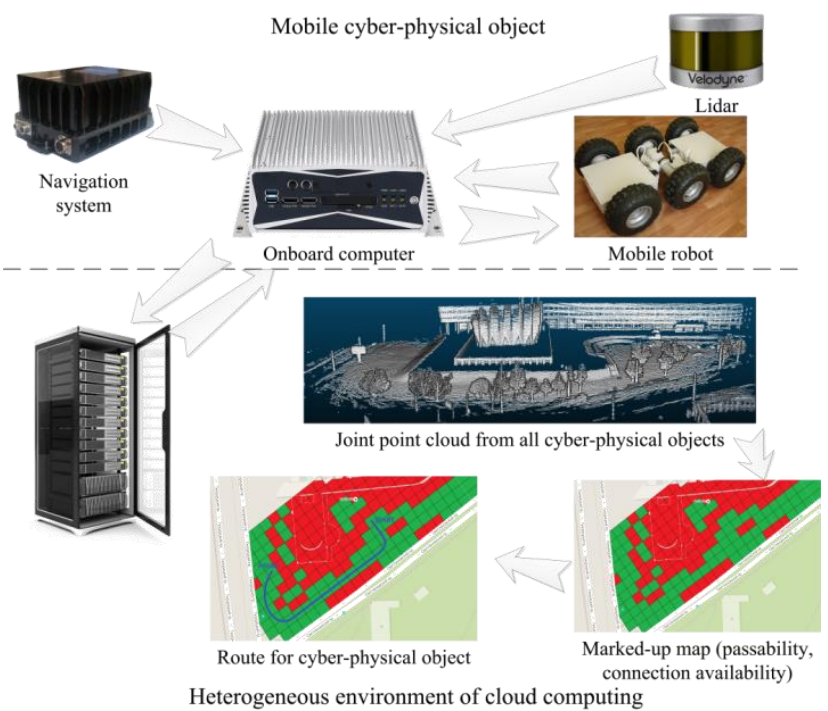

Figure 5. An example of using the cloud environment to control a group of robots: constructing joint map

Network-centric control system can be considered as data flows from various agents to their avatar applications. In our works (Ilyashenko et al., 2014; Ilyashenko et al., 2015; Muliukha et al., 2015) we consider the preemptive priority queueing system with two classes of packets. The packets of class 1 (2) arrive into the buffer according to the Poisson process with rate $\lambda_{1}\left(\lambda_{2}\right)$. The service time has the exponential distribution with the same rate $\mu$ for each class. The service times are independent of the arrival processes. The buffer has a finite size $k \quad(1<k<\infty)$ and it is shared by both types of customers. The absolute priority in service is given packets of the first class. Unlike typical priority queueing considered system is supplied by the randomized push-out mechanism. If the buffer is full, a new coming customer of class 1 can push out of the buffer a customer of class 2 with the probability $\alpha$. Note that if $\alpha=1$ we retrieve the standard non-randomized push-out.

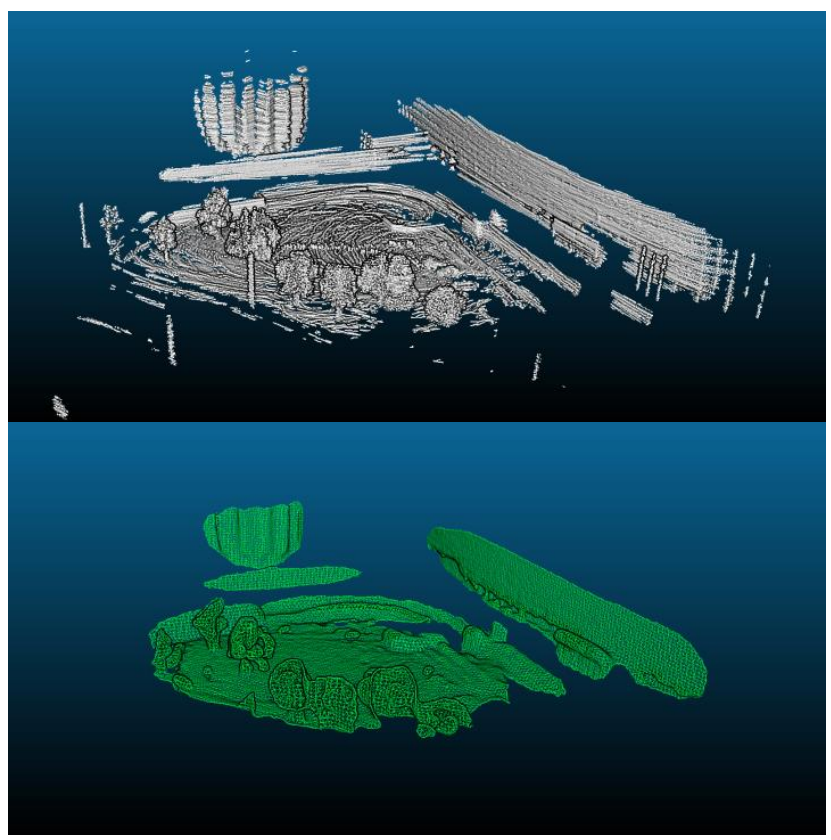

Figure 6. An example of the point cloud vectorization using PCL library

The summarized entering stream will be the elementary with intensity $\lambda=\lambda_{1}+\lambda_{2}$. If we'll trace only the general number of packets in system, then simplified one-data-flow model would be $M / M / 1 / k$ type. The special modification of standard Kendel notation intended for priority queueing was proposed by G.P.Basharin. In the modified system the general structure of a label and sense of its separate positions remains however in each position the vectorial symbolic is used. There is an additional symbol $f_{i}^{j}$, where $i$ specifies priority type $(0-$ without a priority, 1 - relative, 2 -absolute), and $j$ specifies a type of the pushing out mechanism ( 0 - without pushing out, 2 the determined pushing out).

We have analyzed received data and concluded that the computer network is modelled by means of queueing with the finite buffer size and the probability push-out mechanism in a combination with an absolute priority. The offered effective computing algorithm allows network engineers and designers to estimate possible variants of network traffic load. In overload networks, then $\lambda=\lambda_{1}+\lambda_{2} \gg \mu$, the dependence of loss priority from variable $\alpha$ is not linear and even not monotonous function. In that case the network with an absolute priority gives a maximum of advantages to the most important types of packets. Our algorithm and network model is flexible and in comparison with a relative priority. 
Queuing theory describes the process of information exchange in a network-centric robot group control system and ensures the required quality of service for each information flow between the agent and its virtual avatar. The proposed model for information exchange was used in practice in the framework of space experiments Kontur and Kontur-2 (Zaborovsky et al., 2015).

\section{Conclusions}

This paper proposes a network-centric approach to the description of the control system for a group of mobile robots.

Under this approach, each mobile robot is regarded as cyber-physical object, consisting of a mobile agent, and a virtual avatar that operates in a high performance heterogeneous cloud.

The use of network-centric approach allows to take an advantage of the combination of multi-agent and centralized control.

A necessary condition for the functioning of such a control system is the availability of reliable and highspeed communication channels between the agent and its avatar.

The reliability of these channels is ensured by using heterogeneous networks (LTE / Wi-Fi / 3G and etc.).

The required level of quality of service provided by prioritizing traffic based on the model of queuing theory.

\section{Acknowledgment}

This research was supported by RFBR grant № 15-29-07131 ofi_m.

\section{References}

A. Ilyashenko, O. Zayats, V. Muliukha, and L. Laboshin. Further Investigations of the Priority Queuing System with Preemptive Priority and Randomized Push-out Mechanism. Lecture Notes in Computer Science 8638: 433-443, 2014

A. Ilyashenko, O. Zayats, V. Muliukha, and A. Lukashin. Alternating Priorities Queueing System with Randomized Push-out Mechanism. Lecture Notes in Computer Science 9247: 436-445, 2015. doi: 10.1007/978-3-319-231266_38.

B. Kehoe, S. Patil, P. Abbeel, and K. Goldberg. A Survey of Research on Cloud Robotics and Automation. IEEE Transactions on Automation Science and Engineering, 12(2): 398-409, 2015

V. Muliukha, A. Ilyashenko, O. Zayats, and V. Zaborovsky. Preemptive Queueing System with Randomized Push-out Mechanism. Communications in Nonlinear Science and Numerical Simulation 21(1-3): 147-158, 2015

J.A. Wheeler. Information, Physics, Quantum: the Search for Links. In Proceedings III International Symposium on Foundations of Quantum Mechanics, Tokyo, pages 354368,1989
V. Zaborovsky, M. Guk, V. Muliukha, and A. Ilyashenko. Cyber-Physical Approach to the Network-Centric Robot Control Problems. Lecture Notes in Computer Science 8638: 619-629, 2014

V. Zaborovsky, V. Muliukha, and A. Ilyashenko. CyberPhysical Approach in a Series of Space Experiments "Kontur". Lecture Notes in Computer Science 9247: 745758, 2015. doi: 10.1007/978-3-319-23126-6_69 